\title{
Front Matter: Volume 8455
}

, "Front Matter: Volume 8455," Proc. SPIE 8455, Metamaterials: Fundamentals and Applications V, 845501 (15 October 2012); doi: 10.1117/12.2011531

SPIE Event: SPIE NanoScience + Engineering, 2012, San Diego, California, United SPIE. States 


\section{PROCEEDINGS OF SPIE}

\section{Metamaterials: Fundamentals and Applications $V$}

Allan D. Boardman

Nader Engheta

Mikhail A. Noginov

Nikolay I. Zheludev

Editors

12-16 August 2012

San Diego, California, United States

Sponsored and Published by

SPIE 
The papers included in this volume were part of the technical conference cited on the cover and title page. Papers were selected and subject to review by the editors and conference program committee. Some conference presentations may not be available for publication. The papers published in these proceedings reflect the work and thoughts of the authors and are published herein as submitted. The publisher is not responsible for the validity of the information or for any outcomes resulting from reliance thereon.

Please use the following format to cite material from this book:

Author(s), "Title of Paper," in Metamaterials: Fundamentals and Applications V, edited by Allan D. Boardman, Nader Engheta, Mikhail A. Noginov, Nikolay I. Zheludev, Proceedings of SPIE Vol. 8455 (SPIE, Bellingham, WA, 2012) Article CID Number.

ISSN: 0277-786X

ISBN: 9780819491725

Published by

SPIE

P.O. Box 10, Bellingham, Washington 98227-0010 USA

Telephone +1 3606763290 (Pacific Time) · Fax +1 3606471445

SPIE.org

Copyright (@ 2012, Society of Photo-Optical Instrumentation Engineers.

Copying of material in this book for internal or personal use, or for the internal or personal use of specific clients, beyond the fair use provisions granted by the U.S. Copyright Law is authorized by SPIE subject to payment of copying fees. The Transactional Reporting Service base fee for this volume is $\$ 18.00$ per article (or portion thereof), which should be paid directly to the Copyright Clearance Center (CCC), 222 Rosewood Drive, Danvers, MA 01923. Payment may also be made electronically through CCC Online at copyright.com. Other copying for republication, resale, advertising or promotion, or any form of systematic or multiple reproduction of any material in this book is prohibited except with permission in writing from the publisher. The CCC fee code is 0277-786X/12/\$18.00.

Printed in the United States of America.

Publication of record for individual papers is online in the SPIE Digital Library.

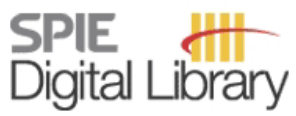

SPIEDigitalLibrary.org

Paper Numbering: Proceedings of SPIE follow an e-First publication model, with papers published first online and then in print and on CD-ROM. Papers are published as they are submitted and meet publication criteria. A unique, consistent, permanent citation identifier (CID) number is assigned to each article at the time of the first publication. Utilization of CIDs allows articles to be fully citable as soon as they are published online, and connects the same identifier to all online, print, and electronic versions of the publication. SPIE uses a six-digit CID article numbering system in which:

- The first four digits correspond to the SPIE volume number.

- The last two digits indicate publication order within the volume using a Base 36 numbering

system employing both numerals and letters. These two-number sets start with 00, 01, 02, 03, 04, $05,06,07,08,09,0 A, 0 B \ldots 0 Z$, followed by 10-1Z, 20-2Z, etc.

The CID Number appears on each page of the manuscript. The complete citation is used on the first page, and an abbreviated version on subsequent pages. Numbers in the index correspond to the last two digits of the six-digit CID Number. 


\section{Contents}

vii Conference Committee

HYPERBOLIC METAMATERIALS I

845507 Hyperbolic metamaterials platforms for tuning reflectance, transmittance and absorption [8455-6]

T. U. Tumkur, J. K. Kitur, L. Gu, B. Chu, Norfolk State Univ. (United States); V. A. Podolskiy, Univ. of Massachusetts Lowell (United States); E. E. Narimanov, Purdue Univ. (United States); M. A. Noginov, Norfolk State Univ. (United States)

NONLINEAR METAMATERIALS I

8455 OB Nonlinear and active RF metamaterial applications using embedded devices [8455-11]

A. R. Katko, A. M. Hawkes, S. A. Cummer, Duke Univ. (United States)

\section{PLASMONIC METAMATERIALS I}

8455 OK Surface-plasmon holography with white light illumination [8455-20]

M. Ozaki, RIKEN (Japan) and Tokyo Denki Univ. (Japan); J. Kato, RIKEN (Japan); S. Kawata, RIKEN (Japan) and Osaka Univ. (Japan)

HYPERBOLIC METAMATERIALS II

$8455 \mathrm{OV}$ Effects of nonlocal response on the density of states of hyperbolic metamaterials [8455-25]

W. Yan, M. Wubs, N. A. Mortensen, Technical Univ. of Denmark (Denmark)

\section{NOVEL TRENDS AND CONCEPTS}

845510 Repulsive Casimir Force using metamaterials [8455-30]

V. K. Pappakrishnan, P. C. Mundru, D. A. Genov, Louisiana Tech Univ. (United States)

845511 Radiation pressure and photon momentum in negative-index media [8455-31]

M. Mansuripur, College of Optical Sciences, The Univ. of Arizona (United States);

A. R. Zakharian, Corning Inc. (United States)

845512 Trouble with the Lorentz law of force: response to critics [8455-32]

M. Mansuripur, College of Optical Sciences, The Univ. of Arizona (United States) 
845514 Realization of metamaterial structures by non-lithographic processes (Invited Paper) [8455-33]

A. Piqué, N. A. Charipar, H. Kim, M. A. Kirleis, R. C. Y. Auyeung, U.S. Naval Research Lab. (United States); A. T. Smith, Nova Research, Inc. (United States); K. M. Metkus, U.S. Naval Research Lab. (United States); S. A. Mathews, U.S. Naval Research Lab. (United States)

\section{MAGNETIC METAMATERIALS}

8455 1D Modification of electric and magnetic dipole emission in anisotropic plasmonic systems [8455-41]

N. Noginova, R. Hussain, M. A. Noginov, Norfolk State Univ. (United States); J. Vella, Air Force Research Lab. (United States) and General Dynamics Information Technology (United States); A. Urbas, Air Force Research Lab. (United States)

$8455 \mathrm{IF}$ Negative refractive index in ferromagnetic semiconductors: experimental verification [8455-43]

A.-G. Kussow, Y. Ait El Aoud, H. M. Jaradat, A. Akyurtlu, Univ. of Massachusetts Lowell (United States)

8455 IG Bottom-up metamaterials with an isotropic magnetic response in the visible [8455-44] S. Mühlig, Friedrich-Schiller-Univ. Jena (Germany); J. Dintinger, Ecole Polytechnique Fédérale de Lausanne (Switzerland); A. Cunningham, Univ. de Genèva (Switzerland); T. Scharf, Ecole Polytechnique Fédérale de Lausanne (Switzerland); T. Bürgi, Univ. de Genèva (Switzerland); C. Rockstuhl, F. Lederer, Friedrich-Schiller-Univ. Jena (Germany)

\section{METAMATERIALS LENSES AND FOCUSING}

$84551 \mathrm{~K}$ Super-resolution for a point source using positive refraction [8455-48]

J. C. Miñano, P. Benítez, J. C. González, D. Grabovičkić, H. Ahmadpanahi, Univ. Politécnica de Madrid (Spain)

\section{NIMS, CLOAKS, AND TRANSFORMATION OPTICS}

8455 IP An innovative cloak enables arbitrary multi-objects hidden with visions and movements [8455-53]

T.-Y. Huang, H.-C. Lee, I.-W. Un, T.-J. Yen, National Tsing Hua Univ. (Taiwan)

$84551 Q \quad$ Flexible zero refractive index optical metamaterial with matched impedance [8455-54]

S. Yun, L. Lin, Z. H. Jiang, D. Ma, Z. Liu, D. H. Werner, T. S. Mayer, The Pennsylvania State Univ. (United States) 
$84551 \mathrm{U}$ Isotropic and anisotropic continuous index photon traps based on composite optical materials [8455-58]

D. A. Genov, Louisiana Tech Univ. (United States)

THEORY AND MODELING

845529 Anderson localization effects via the invariant measure for discretely disordered negative and positive index structures [8455-74]

G. J. Kissel, Univ. of Southern Indiana (United States)

8455 2B Optical functions of nanocrystalline $\mathrm{ZnO}$ containing voids and doped with Ga [8455-76] A. V. Gavrilenko, K. Dondapati, V. I. Gavrilenko, Norfolk State Univ. (United States); J. Kim, G. V. Naik, A. Boltasseva, Purdue Univ. (United States)

\section{PLASMONIC METAMATERIALS II}

$84552 \mathrm{H} \quad$ Plasmon induced transparency in cascaded $\pi$-shaped structures [8455-82]

A. E. Cetin, A. Artar, M. Turkmen, A. A. Yanik, H. Altug, Boston Univ. (United States)

NONLINEAR METAMATERIALS II

845520 Tunable and nonlinear fishnet metamaterials based on liquid crystal infiltration (Invited Paper) [8455-89]

S. Kruk, A. Minovich, J. Farnell, I. McKerracher, F. Karouta, J. Tian, D. A. Powell, I. V. Shadrivov, H. H. Tan, C. Jagadish, D. N. Neshev, Y. S. Kivshar, The Australian National Univ. (Australia)

$84552 Q \quad$ Active nonlinear metamaterials loaded with negative differential resistance elements and circuits [8455-91]

J. P. Barrett, S. A. Cummer, Duke Univ. (United States)

\section{POSTER SESSION}

$84552 Y \quad$ Interaction of low-energy electronic excitation of $\mathrm{Tm}^{3+}$ ions with acoustic vibrations in crystal $\mathrm{KTm}\left(\mathrm{MoO}_{4}\right)_{2}$ [8455-99]

S. N. Poperezhai, B. Verkin Institute for Low Temperature Physics and Engineering (Ukraine);

D. L. Kamenskyi, Forschungszentrum Dresden-Rossendorf (Germany); V. I. Kut'ko, B. Verkin

Institute for Low Temperature Physics and Engineering (Ukraine)

845530 The invariant measure for Anderson localized negative index metamaterials continuously disordered [8455-101]

G. J. Kissel, A. Williams, Univ. of Southern Indiana (United States) 
845531 Magnetic nanoparticles for tunable microwave metamaterials [8455-103]

N. Noginova, Q. L. Williams, Norfolk State Univ. (United States); P. Dallas, E. P. Giannelis, Cornell Univ. (United States)

845532 Nonlinear cloaking at microwave frequencies [8455-104]

E. A. Gurvitz, E. A. Sedykh, M. K. Khodzitskiy, National Research Univ. of Information Technologies, Mechanics and Optics (Russian Federation)

Author Index 


\section{Conference Committee}

Symposium Chairs

David L. Andrews, University of East Anglia Norwich (United Kingdom) James G. Grote, Air Force Research Laboratory (United States)

Symposium Cochairs

Satoshi Kawata, Osaka University (Japan)

Manijeh Razeghi, Northwestern University (United States)

Conference Chairs

Allan D. Boardman, University of Salford (United Kingdom) Nader Engheta, University of Pennsylvania (United States)

Mikhail A. Noginov, Norfolk State University (United States)

Nikolay I. Zheludev, University of Southampton (United Kingdom)

\section{Conference Program Committee}

David L. Andrews, University of East Anglia Norwich (United Kingdom) Koray Aydin, Northwestern University (United States)

Rola Aylo, University of Dayton (United States)

Igal Brener, Sandia National Laboratories (United States)

Larry R. Dalton, University of Washington (United States)

Ildar R. Gabitov, The University of Arizona (United States)

F. Javier García de Abajo, Consejo Superior de Investigaciones

Científicas (Spain)

Satoshi Kawata, Osaka University (Japan)

Jacob B. Khurgin, The Johns Hopkins University (United States)

Yuri S. Kivshar, The Australian National University (Australia)

Joachim R. Krenn, Karl-Franzens-Universität Graz (Austria)

Akhlesh Lakhtakia, The Pennsylvania State University (United States)

Ulf Leonhardt, University of St. Andrews (United Kingdom)

Natalia M. Litchinitser, University at Buffalo (United States)

Martin W. McCall, Imperial College London (United Kingdom)

Martin Moskovits, API Nanotronics Corporation (United States) and University of California, Santa Barbara (United States)

Evgenii E. Narimanov, Purdue University (United States)

Vladimir M. Shalaev, Purdue University (United States)

Gennady B. Shvets, The University of Texas at Austin (United States)

David R. Smith, Duke University (United States)

Costas M. Soukoulis, lowa State University (United States)

Mark I. Stockman, Georgia State University (United States) 
Sergei Tretyakov, Aalto University School of Science and Technology (Finland)

Din Ping Tsai, National Taiwan University (Taiwan)

Augustine M. Urbas, Air Force Research Laboratory (United States)

Martin Wegener, Karlsruher Institut für Technologie (Germany)

\section{Session Chairs}

1 Nanoantennas

Mark Brongersma, Geballe Laboratory for Advanced Materials (GLAM) (United States)

2 Hyperbolic Metamaterials I

Martin Wegener, Karlsruher Institut für Technologie (Germany)

3 Nonlinear Metamaterials I

Jacob B. Khurgin, Johns Hopkins University (United States)

4 Plasmonic Metamaterials I

Nikolay I. Zheludev, University of Southampton (United Kingdom)

5 Hyperbolic Metamaterials II

David R. Smith, Duke University (United States)

6 Novel Trends and Concepts

Ekaterina Poutrina, Duke University (United States)

$7 \quad$ Making Metamaterials

Rashid Zia, Brown University (United States)

8 Magnetic Metamaterials

Uriel Levy, The Hebrew University of Jerusalem (Israel)

9 Metamaterials Lenses and Focusing

Jason Gage Valentine, Vanderbilt University (United States)

10 NIMs, Cloaks, and Transformation Optics

Xingjie Ni, Purdue University (United States)

11 Metamaterial Absorbers

Costas M. Soukoulis, lowa State University (United States)

12 Gain and Stimulated Emission

F. Javier García de Abajo, Consejo Superior de Investigaciones Científicas (Spain) 
13 Chirality and Vortixes

Fumin Huang, Guangdong University of Technology (China)

14 Theory and Modeling

Natalia M. Litchinitser, University at Buffalo (United States)

15 Plasmonic Metamaterials II

Ekmel Özbay, Bilkent University (Turkey)

16 Applications of Metamaterials

Dragomir Neshev, The Australian National University (Australia)

17 Nonlinear Metamaterials II

Augustine Urbas, Air Force Research Laboratory (United States)

18 Active and Tunable Metamaterials

Viktor A. Podolskiy, University of Massachusetts Lowell (United States) 
Proc. of SPIE Vol. $8455845501-10$

Downloaded From: https://www.spiedigitallibrary.org/conference-proceedings-of-spie on 26 Apr 2023 Terms of Use: https://www.spiedigitallibrary.org/terms-of-use 